\title{
Filtering Methods for Error Reduction in Spacecraft Attitude Estimation using Quaternion Star Trackers
}

\author{
Philip C. Calhoun ${ }^{1}$ \\ NASA Goddard Space Flight Center, Greenbelt, Md., 2077 I \\ Joseph E. Sedlak ${ }^{2}$ and Emil Superfin ${ }^{3}$ \\ a.i. solutions, Inc., Lanham, MD, 20706
}

\begin{abstract}
Precision attitude determination for recent and planned space missions typically includes quaternion star trackers (ST) and a three-axis inertial reference unit (IRU). Sensor selection is based on estimates of knowledge accuracy attainable from a Kalman filter (KF), which provides the optimal solution for the case of linear dynamics with measurement and process errors characterized by random Gaussian noise with white spectrum. Non-Gaussian systematic errors in quaternion STs are often quite large and have an unpredictable timevarying nature, particularly when used in non-inertial pointing applications. Two filtering methods are proposed to reduce the attitude estimation error resulting from ST systematic errors, 1) extended Kalman filter (EKF) augmented with Markov states, 2) Unscented Kalman filter (UKF) with a periodic measurement model. Realistic assessments of the attitude estimation performance gains are demonstrated with both simulation and fight telemetry data from the Lunar Reconnaissance Orbiter.
\end{abstract}

\section{Introduction}

$\mathrm{P}$ recision attitude determination for recent and planned space missions typically includes quaternion star trackers (ST) and three-axis inertial reference units (IRU). Sensor selection is based on estimates of knowledge accuracy attainable from a Kalman filter (KF), which provides the optimal solution for the case of linear dynamics and measurement and process errors characterized by random Gaussian noise with white spectrum. Many near term future missions will utilize commercial-off-the-shelf (COTS) quaternion STs with systematic errors of approximately 0.5 arc-min ( 3 sigma). This is primarily due to the availability of COTS sensors with reasonable cost in this performance range. Missions that need more precise knowledge will require higher cost, or perhaps custom designed, star trackers or fine guidance sensors to meet performance objectives. When COTS quaternion STs are combined with navigation grade IRUs, a typical EKF reduces effects of random errors on the overall attitude knowledge to a few arcseconds. However, the effect of ST systematic errors are often much larger and are not attenuated by the 6 -state attitude extended Kalman filter (EKF) ${ }^{1}$. These errors, resulting from inaccuracies in individual star measurements, are primarily evident along the ST boresight for narrow field of view (FOV) STs. ${ }^{2,3}$ They are the ciontiuant item in a typical spacecraft attitude knowledge error analysis.

The effects of ST systematic errors on quaternion ST solutions are characterized by an unpredictable timevarying error, particularly when used in non-inertial pointing applications. As tracked stars move across the FOV spatial errors become time varying. Since the ST quaternion output is an aggregate of individual star measurements acquired at various locations on the ST focal plane, systematic changes in the output error mean and variance are evident as stars dynamically transverse the FOV. For a typical nadir-pointing spacecraft in low Earth orbit (period $90 \mathrm{~min}$ ) stars would traverse a typical ST FOV (15 deg) within $3.75 \mathrm{~min}$. Thus, any ST focal plane or other spatial FOV errors would appear as time-varying systematic errors with time scales of a few minutes. Since most COTS STs have relatively narrow FOV size these errors are primarily evident about the ST boresight direction. For example, a typical quaternion ST with transverse systematic errors of a few arcseconds would have $\sim 0.5$ arc-min systematic errors about the ST boresight.

\footnotetext{
${ }^{1}$ Senior Aerospace Engineer, Attitude Control System Engineering Branch, MS 591, 8800 Greenbelt Rd., Greenbelt, Md. 20771, AlAA member.

${ }^{2}$ Senior Aerospace Engineer, a.i. solutions, Lanham, Md. 20706.

${ }^{3}$ Aerospace Engineer, a.i. solutions, Lanham, Md. 20706.
} 
An example, used to illustrate typical ST systematic error, is presented from Lunar Reconnaissance Orbiter (LRO) flight telemetry. The LRO attitude determination system employs two quaternion STs and a single three-axis IRU. ${ }^{4}$ The two ST boresights are separated by approximately $60 \mathrm{deg}$. Figure 1 shows a comparison of the typical quaternion output for ST1 to that from ST2 taken from LRO telemetry. The residual attitude knowledge errors are small angle approximations to the delta quaternion computed from the output of the STs. These residuals are then filtered, using a low pass filter with bandwidth of $1 \mathrm{cycle} / \mathrm{min}$, to remove the high frequency random error. During this period LRO was nadir-pointing in low lunar orbit (period $\sim 113 \mathrm{~min}$ ). Since each ST has a 16-deg FOV, the stars traverse the FOV in approximately $5 \mathrm{~min}$. Significant non-white systematic errors, with periods from $2-10$ $\mathrm{min}$, are clearly present in this data. There are errors with larger time scale which are primarily attributed to thermal alignment effects as the STs are exposed to varying solar heating conditions along the orbit. The objective of this study is to remove the larger short term systematic error associated with stars traversing the FOV.

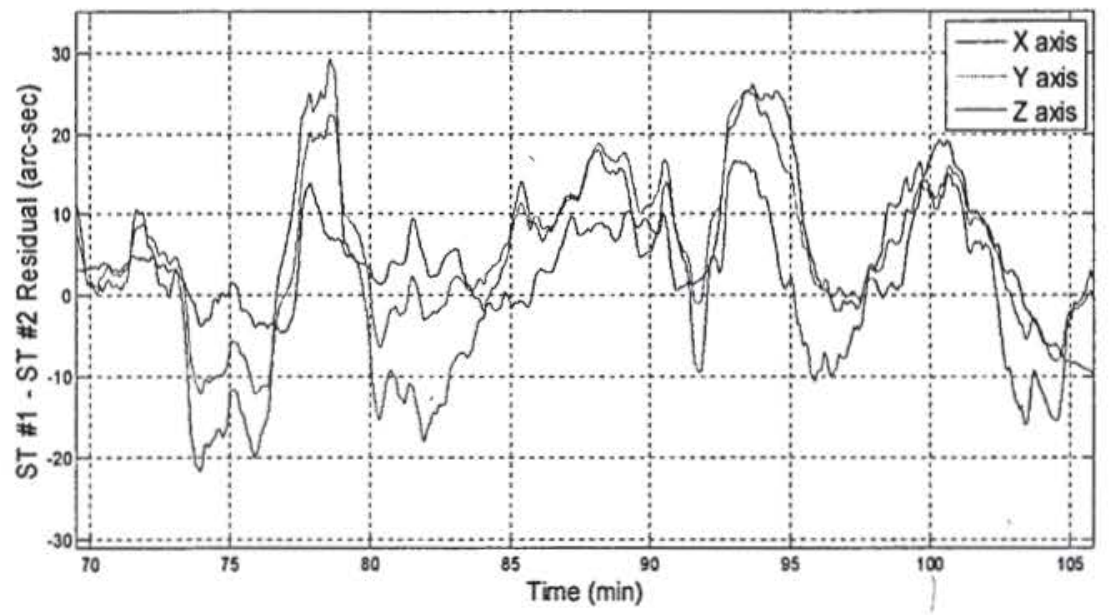

Figure 1. LRO ST \#1 minus ST \#2 Residuals, low pass filtered (T=1 min).

Extensive works in the open literature are available on the subject of spacecraft attitude estimation. Early papers include the well known development of the 6-state attitude EKF. ${ }^{\prime}$ A very good recently published survey on spacecraft attitude estimation documents a variety of nonlinear methods applied to state-of-the-art attitude estimation. ${ }^{5}$ However, few of the published works address systematic measurement errors of the type described in this paper. Haley ${ }^{3}$ and Haley et al. ${ }^{2}$ identify the nature and basis of ST systematic error, providing also an illustration of their effect on precision attitude determination. They include a list of potential approaches to reduce the effects of ST systematic error on the attitude determination performance. Reference 2 mentions an ongoing effort to model the systematic error using a first-order Markov process, similar to the method described in Section II below. However, this work does not include specific algorithmic development, implementation, and results to demonstrate potential performance improvement. Sedlak and Hashmall ${ }^{6}$ develop a first order Markov state augmentation for describing systematic errors it magnetometer measurements for attitudy zstimation using a standard Kalman filter approach. The algorithmic work developed therein will serve as the motivation for a similar approach to model the ST systematic error with a Markov state, as described in Section II.

In this study we seek to develop filtering methods to reduce the effect of ST systematic errors on the overall attitude knowledge to a level equivalent to errors associated with ST random errors. This will enable higher performance applications for a given attitude sensor complement or provide for potential hardware cost reduction in achieving particular knowledge stability specifications. Two methods for reducing the knowledge errors resulting from systematic ST errors are developed in this paper. The first method, described in Section II, is a 6-state attitude EKF where the state vector is augmented with first order Markov terms that represent the ST systematic errors. This is similar to the methods employed for reduction of systematic magnetometer errors in attitude estimation. ${ }^{5}$ The second method, described in Section III, is an Unscented Kalman filter (UKF) utilizing a time-varying measurement model. In this filter implementation, the ST systematic errors are modeled as a series of periodic basis functions similar to a Fourier series representation. This method is based on a paper that describes the use of this type of measurement model in the reduction of periodic errors in measurement processes for rotating machinery. ${ }^{7}$ The filter states are augmented with bias states to estimate the amplitudes associated with each basis function frequency (i.e., Fourier series coefficients). An estimate of the systematic error is formed as the summation of the periodic terms, 
which is used to adjust the measurement residual accordingly. Both of these methods utilize the multiplicative form of the attitude measurement residual, as in the traditional 6-state EKF.'

Realistic assessments of performance gains attained by each of the two proposed filtering methods are demonstrated by exercising them with simulated measurement data, as well as flight telemetry data from the LRO, as described in Section IV. The systematic errors associated with each ST are assumed uncorrelated from each other. Thus, the resulting attitude estimates, associated with processing measurements from both STs simultaneously in a 6-state EKF, should provide a reasonable baseline for determination of the relative performance improvements of each proposed method.

\section{Method 1: Extended Kalman Filter with Markov States for ST errors}

This section describes a modification of the standard quaternion plus gyro bias 6-state EKF typically used for spacecraft attitude determination. The state is augmented with a scalar correction term representing a rotation about the boresight of a single autonomous star tracker (ST). For simplicity, only a single ST correction is modeled in the tests presented in this work; however, in practice, one will have a separate scalar correction for each ST. Subsection A. describes the ST boresight rotation angle as a first-order Markov model. Subsection B presents the ST sensor model with the boresight rotation correction and gives the derivation of the sensitivity matrix, $H$.

\section{A. State Augmentation using Markov States for Systematic ST errors}

The unaugmented state vector includes just the attitude quaternion and the gyro bias. The corresponding error state is

$$
x=\left[\begin{array}{c}
\vec{a} \\
\Delta \vec{b}
\end{array}\right],
$$

where $\vec{a}$ is the attitude error vector, with norm $\alpha$, and $\Delta \vec{b}$ is the gyro bias error vector. The true quaternion, $q_{t r u v}$, and bias, $\vec{b}_{\text {true }}$, are related to the error state and the current state estimate by

$$
\begin{gathered}
q_{\text {true }}=\left[\begin{array}{c}
-\hat{\alpha} \sin \left(\frac{\alpha}{2}\right) \\
\cos \left(\frac{\alpha}{2}\right)
\end{array}\right] \otimes q_{e s t} \equiv d q_{a}^{*} \otimes q_{e s t} \\
\vec{b}_{\text {true }}=\vec{b}_{e s t}+\Delta \vec{b} .
\end{gathered}
$$

The quaternion product convention used here is that quaternions multiply in the same order as direction-cosine matrices, and is denoted by the symbol $\otimes$. The sign convention in Eq.(2) simplifies some signs that appear elsewhere in the EKF but may differ from the signs used for the attitude error elsewhere in the literature. Caution must be taken with the corresponding signs in the propagation matrix and sensitivity matrix.

The propagation matrix from time $t_{\text {, }}$ to $t_{t+1}$ for the state $x$ can be written

$$
\begin{aligned}
x_{i+1} & =\Phi_{1} x_{i}=\left[\begin{array}{ll}
\varphi_{i} & \psi_{i} \\
0_{3} & I_{3}
\end{array}\right] x_{i}
\end{aligned}
$$

where the attitude and bias transition matrices, $\varphi$ and $\psi$, can be found in [1]. The covariance propagation is given by

$$
P_{i+1}=\Phi_{1} P_{1} \Phi_{i}^{T}+Q_{1}
$$

where $Q_{1}$ is the process noise, depending on the gyro noise sources for accumulated angle random walk and bias random walk.

The augmented state is

$$
x^{a}=\left[\begin{array}{l}
x \\
\theta
\end{array}\right]
$$


where $\theta$ is a correction angle to be applied to the ST measured attitude as a rotation about its boresight. This is described in more detail in the next section. The correction angle, $\theta$, is assumed to satisfy the equation

$$
\frac{d}{d t} \theta=-\beta \theta+u(t),
$$

where $\beta=1 / \tau$ is the inverse Markov time constant, and $u(t)$ is Gaussian-distributed white noise. The discrete time solution to Eq.(7) is

$$
\theta_{i+1}=\Psi_{i} \theta_{i}+u_{i}
$$

where

$$
\Psi\left(t_{i+1}, t_{i}\right)=\Psi_{i}=e^{-\beta \Delta t_{i}},
$$

with $\Delta t_{t}=t_{t+1}-t_{t}$, and where

$$
u_{i}=\int_{t_{i}}^{t_{i+1}} \Psi\left(t_{i+1}, \tau\right) u(\tau) d \tau
$$

(see, e.g., Gelb [10]). Then, the expected augmented state propagation is written as

$$
x_{i+1}^{a}=\Phi_{i}^{a} x_{i}^{a}=\left[\begin{array}{cc}
\Phi_{i} & 0_{6,1} \\
0_{1,6} & \Psi_{i}
\end{array}\right] x_{i}^{a} .
$$

The covariance, $p_{\theta}$, of angle $\theta$ satisfies the equation

$$
\dot{p}_{\theta}(t)=-\beta p_{\theta}-p_{\theta} \beta+Q_{\theta}(t),
$$

where

$$
E\left[u(t) u\left(t^{\prime}\right)\right]=Q_{\theta}(t) \delta\left(t-t^{\prime}\right) .
$$

Assuming the process noise $Q_{\theta}(t)$ is constant, it is convenient to assume that the covariance of the boresight rotation correction is also constant, $\dot{p}_{\theta}(t)=0$. Eq.(12) then places a condition on the initial (and constant) covariance

$$
p_{\theta}=Q_{\theta} /(2 \beta),
$$

which makes one fewer tuning parameter to adjust. The covarianse can be written as the ssuare of the boresight rotation uncertainty angle

$$
p_{\theta}=\sigma_{\theta}^{2} .
$$

Next, the discrete time process noise can be derived by evaluating E[ $\left.u_{l} u_{l}\right]$. Using Eqs.(10) and (13), the first integral is trivial due to the Dirac $\delta$-function, and the second integral yields

$$
Q_{\theta, i}=\sigma_{\theta}^{2}\left(1-\exp \left(-2 \beta \Delta t_{i}\right)\right)
$$

Thus, the augmented state covariance propagation is

$$
P_{i+1}^{a}=\Phi_{i}^{a} P_{i}^{a} \Phi_{i}^{a T}+Q_{i}^{a}, \quad \ldots
$$


where

$$
Q_{i}^{a}=\left[\begin{array}{cc}
Q_{i} & 0 \\
0 & Q_{\theta, i}
\end{array}\right]
$$

\section{B. Star Tracker Measurement Model}

The ST returns a quaternion representing the transformation from the inertial frame to the sensor frame, and thence to the body frame using the ST alignment matrix. This inertial-to-body quaternion will be referred to as $q_{\text {obs }}$. The boresight correction angle described in the previous section is applied to the observed quaternion to obtain an adjusted quaternion,

$$
q_{a d j}=q_{b} \otimes q_{o b s},
$$

where

$$
q_{b}=\left[\begin{array}{c}
\hat{S} \sin \left(\frac{\theta}{2}\right) \\
\cos \left(\frac{\theta}{2}\right)
\end{array}\right],
$$

and with $\hat{S}$ the ST boresight unit vector in the body frame. The ST correction error state, $\Delta \theta$, is related to the truth state and its current estimate as

$$
\theta_{\text {true }}=\theta_{\text {est }}+\Delta \theta
$$

The corresponding quaternion error correction is

$$
d q_{b} \equiv\left[\begin{array}{l}
\hat{S} \sin \left(\frac{\Delta \theta}{2}\right) \\
\cos \left(\frac{\Delta \theta}{2}\right)
\end{array}\right]
$$

so that

$$
d q_{b} \otimes q_{a d j}=q_{\text {true }}+\text { noise } .
$$

The ST residual vector is defined here to be twice the vector part of the quaternion product, $d q$, of the current attitude estimate and the conjugate adjusted observation. Making use of Eqs.(2) and (23), this is

$$
\begin{gathered}
d q \equiv q_{o s t} \otimes q_{a d j}^{*}=d q_{a} \otimes q_{t r u e} \otimes q_{a d j}^{*} \cong d q_{a} \otimes d q_{b} \otimes q_{a d j} \otimes q_{a d j}^{*}=d q_{a} \otimes d q_{b .2} \\
\cong\left[\begin{array}{c}
\frac{\alpha}{2} \hat{\alpha}+\frac{\Delta \theta}{2} \hat{s} \\
1
\end{array}\right]
\end{gathered}
$$

This yields the residual

$$
r e s=\vec{\alpha}+\Delta \theta \hat{S}
$$

Finally, the partial derivatives with respect to the error state components are

$$
\frac{\partial r e s}{\partial \vec{\alpha}}=I_{3 \times 3}, \quad \frac{\partial r e s}{\partial \Lambda \vec{h}}=0_{3 \times 3}, \quad \frac{\partial r e s}{\partial \Lambda A}=\hat{S} .
$$

Thus, the sensitivity matrix is 


$$
H=\left[\begin{array}{lll}
I_{3 \times 3} & 0_{3 \times 3} & \hat{S}
\end{array}\right] .
$$

\section{Method 2: Unscented Kalman Filter with Periodic Measurement Model}

An approach similar to the modification of the standard quaternion plus gyro bias filter described in Section II is presented in this section. However, in this case the state is augmented with a set of bias states that represent the amplitudes of a set of periodic basis functions. This method was used by Wang and Li to augment a Kalman filter for estimation of systematic errors in rotating machinery. ${ }^{7}$ The use of sine and cosine basis functions is motivated by a Fourier series representation of the periodic systematic errors present in the sensor system. For this application the ST systematic errors are nearly periodic since the star field pattern nearly repeats at orbit period. Otherwise, the time-varying nature of the errors is somewhat unpredictable. For non-inertial pointing applications, tracked stars move across the ST FOV and spatial FOV errors result in time varying error in the output quaternion. Since the ST quaternion output is an aggregate of individual star measurements acquired at various locations on the ST focal plane, systematic errors in the quaternion output are evident as stars dynamically traverse the ST FOV. Thus, any ST focal plane or other spatial FOV errors would appear as time-varying systematic errors with time scales of a few minutes, depending on the FOV size.

Subsection A describes the augmented ST measurement model using periodic basis functions. For a set of functions with prior determined fixed frequencies the resulting measurement model is strictly a function of time, not state (i.e., time-varying linear). However, to facilitate the extension of this measurement model to the case of adaptation for basis functions with uncertain, and perhaps varying parameters (e.g., frequency), the UKF method was chosen to implement this estimator. The UKF is a nonlinear estimation method that directly uses the nonlinear system and measurement models for propagation of the state and measurement error covariance matrices, without the linearization errors associated with the EKF. The UKF also has the advantage of using the measurement and state updates of a standard linear Kalman filter, thus allowing ease of implementation of the multiplicative quaternion measurement residual (as developed in Section II B). Subsection B is an overview of the UKF $^{8,9}$ as implemented for this application, based on an algorithm described in [8].

\section{A. Periodic Measurement Model}

Rather than implementing the model for the ST boresight systematic error as a first-order Markov process, as in Eqs. 7-9, the error is expressed in the form of a Fourier series expansion of the true error, where $N_{p}$ is the number of periodic terms in the expansion.

$$
\theta=\sum_{i=1}^{N_{P}}\left(\theta_{P}(2 i-1) * \cos \left(w_{P}(i) * t\right)+\theta_{P}(2 i) * \sin \left(w_{P}(i) * t\right)\right) .
$$

The frequencies associated with the Fourier series basis functions will be assumed as known prior parameters. However, the amplitudes associated with each term are modeled with bias states to facilitate estimation of their time-varying nature. The standard attitude and IRU bias state, given in Eq. (1), is augmented with bias states to form the state model, where $u(t)$ is a white noise input.

$$
x^{a}=\left[\frac{x}{\vec{\theta}_{P}}\right], \quad \frac{d}{d t} \vec{\theta}_{P}=u(t) .
$$

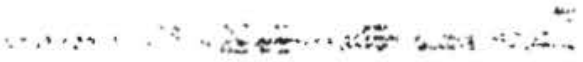

- The state transition matrix for the discrete time implementation of the bias states is then the identity matrix, yielding

$$
\vec{\theta}_{P, l+1}=\vec{\theta}_{P, l} .
$$

This new state vector implies a slightly different measurement model than the one given in Section II B. First, define the multiplicative quaternion measurement residual, $d q_{a}$, using a modified estimate of the current ST quaternion, $q_{S T}$. This estimated ST quaternion, $\hat{q}_{S T}$, is usually given as the IRU propagated prior attitude estimate, $q_{I R U}$. Here $q_{I R U}$ is multiplied by an estimate of the ST boresight systematic error, $q_{B}$, as given by Eq (20), providing 
an appropriate ST systematic error correction. In this case the ST boresight angle error, given by Eq. (28), is substituted for $\theta$ in $\mathrm{Eq}(20)$.

$$
\begin{aligned}
& \hat{q}_{S T}=q_{B} \otimes q_{I T t} \\
& d q_{a}=q_{S T} \otimes \hat{q}_{S T}^{*}
\end{aligned}
$$

The measurement residual can be expressed using small angle approximation for Eq (32) as,

$$
r e s=\vec{a}+\hat{S}\left\{\sum_{i=1}^{N_{P}}\left(\theta_{P}(2 i-1) * \cos \left(w_{P}(i) * t\right)+\theta_{P}(2 i) * \sin \left(w_{P}(i) * t\right)\right)\right\} .
$$

The associated measurement sensitivity matrix is then,

$$
H=\left[I_{3 \times 3} 0_{3 \times 3} \hat{S}\left[\cos \left(w_{P}(i) * t\right), \sin \left(w_{P}(i) * t\right), \ldots, \cos \left(w_{P}\left(N_{P}\right) * t\right), \sin \left(w_{P}\left(N_{P}\right)^{*} t\right)\right]\right] .
$$

Note that this measurement matrix is time-varying with augmented state size equal to $6+{ }^{*} N_{p}$. The model developed herein considers only a single ST systematic error correction; however, in principle, one could have a separate scalar correction for additional STs, which would result in an additional $2 * N_{p}$ states for each additional ST.

Determination of the frequency parameter vector, $w_{p}$, is based on the transit times of the stars across the FOV. An estimate of the typical transit time, $T$, is obtained by assuming each star transits along a circular sector of the ST FOV. The sector length associated with the star transit, $L_{s}$, is a function of the, ST FOV size, $\mathrm{R}_{\text {Fov }}$, and the perpendicular distance from the transit track to the center of the FOV, h. The units for the parameters in Eq. (35) are all given in degrees.

$$
\begin{gathered}
L_{s}=2 * \operatorname{sqrt}\left(\mathrm{R}_{\mathrm{FOV}} \wedge 2-\mathrm{h}^{\wedge} 2\right) \\
T=\left(L_{s} / 360\right)^{*} T_{\text {Orht }}
\end{gathered}
$$

For the LRO application the orbit period $\left(T_{\text {Orbu }}\right)$ is approximately $2 \mathrm{hr}$, and the FOV size is $16 \mathrm{deg}$. Thus, the approximate transit time range is 2.5 to $5 \mathrm{~min}$. Systematic errors resulting from FOV anomalies with circular symmetry with respect to the ST boresight will result in time-varying errors with frequency proportional to the transit time. Asymmetric FOV errors will result in time-varying errors with frequency proportional to twice the transit time. Thus; the dominant systematic error should be within the range of 2.5 to 10 min. Systematic errors within this time range are clearly evident from the LRO ST comparison residuals, shown in Fig. I. Selection of particular frequencies to consider for the basis functions in the periodic measurement model should be consistent with this analysis. The filter with the modified measurement model (Eq. 34) is implemented in a UKF to allow for an easy extension to a nonlinear measurement model. For example, a nonlinear model would result from additional state augmentation for adaptive estimation of the frequency parameters in the measurement model.

\section{B. Unscented Kalman Filter Overview}

This section contains an overview of the UKF algorithms provided in [8] and [9]. Reference 8 provides an excellent introduction to a class of filters called Sigma Point filters, of which the UKF is a member. These filters retain the typical estimator framework associated with optimal linear filtering techniques. The difference is in the method of propagation for the associated error state, measurement, and cross-covariance matrices (i.e., $P_{x r}, P_{y y}$, and $P_{x y}$ in the standard EKF algorithm). A set of so-called sigma points are defined and propagated through the state and measurement models. Then, the covariance matrices are computed numerically from the transformed sigma points.

First, form a set of sigma points, $X_{k-1}$, based on prior state and state error covariance estimates, 


$$
\chi_{k-1}=\left[\hat{x}_{k-1}, \hat{x}_{k-1}+\gamma \sqrt{P_{x x}}, \quad \hat{x}_{k-1}-\gamma \sqrt{P_{x x}}\right]
$$

where $\gamma$ is a tuning parameter defining the spread of the sigma points. These sigma points are then propagated through a potentially nonlinear dynamic state transition model

$$
\vec{\chi}_{k}(i)=f\left(\vec{\chi}_{k-1}(i), t\right) \text {, for } \mathrm{i}=1: 2 \mathrm{~L}
$$

where (i) refers to the ith row vector associated with the sigma points $X$, and $L$ is the number of states. An estimate of the state is then formed as a weighted sum of the sigma points

$$
\hat{x}_{k}=\sum_{i=0}^{2 L} w_{i}^{m} \chi_{k-1}(i)
$$

The post-update state error covariance matrix is then estimated numerically as a weighted sum of the sigma point error states

$$
P_{x x}^{+}=\sum_{i=0}^{2 L} w_{i}^{c}\left(\chi_{k-1}(i)-\hat{x}_{k}\right)\left(\chi_{k-1}(i)-\hat{x}_{k}\right)^{T}
$$

The sigma points are then transformed through the measurement model to form a set of pseudo-measurement estimates based on the sigma points

$$
Y_{k-1}=h\left(\chi_{k-1}(i), t\right)
$$

An estimate of the measurement is then computed numerically as a weighted sum of the pseudo sigma point measurements

$$
\hat{y}_{k}=\sum_{i=0}^{2 L} w_{i}^{m} Y_{k-1}(i)
$$

Finally, the measurement and cross covariance matrices are computed numerically as weighted sums of the associated error state and measurement residuals

$$
\begin{gathered}
\ldots \ldots \ldots \ldots+P_{y y}=\sum_{i=0}^{2 l} w_{i}\left(Y_{k-1}(i)-\hat{y}_{k}\right)\left(Y_{k-1}(i)-\hat{y}_{k}\right), \ldots \ldots \\
P_{x y}=\sum_{i=0}^{2 l} w_{l}^{c}\left(\chi_{k-1}(i)-\hat{x}_{k}\right)\left(Y_{k-1}(i)-\hat{y}_{k}\right)^{T}
\end{gathered}
$$

The UKF implementation has the advantage, over some other nonlinear filtering methods, of preserving the measurement update framework of the EKF. Thus, the multiplicative quaternion residual is utilized in a manner identical to the EKF formulation. In fact, with proper choice of the weighting and tuning factors it can be shown that for a linear system with Gaussian errors, the estimates converge to the optimal filter estimates. A summary of the method for selection of the UKF weighting and tuning parameters is given below, based on algorithms for the scaled unscented transform. In this UKF implementation $\kappa=0, \alpha=0.01$, and $\beta=2$.

$$
\lambda=\alpha^{2}(L+\kappa)-L \quad \gamma=\sqrt{\lambda+L}
$$




$$
\begin{gathered}
w_{0}^{m}=\frac{\lambda}{\lambda+L} \quad w_{0}^{c}=\frac{\lambda}{\lambda+L}\left(1-\alpha^{2}+\beta\right) \\
w_{1}^{m}=w_{t}^{c}=\frac{1}{2(\lambda+L)}, i \neq 0
\end{gathered}
$$

\section{Results}

The performance of the proposed filtering methods was evaluated using simulated data as well as LRO flight telemetry. The simulated data used hueristic models of the ST systematic errors based on observed filter residuals from LRO flight telemetry. This approach provided a reasonable simulation assessment in lieu of a more realistic high fidelity model of specific ST systematic error contributors, such as those described in [2] and [3]. Two models, one a colored noise process and the other a deterministic model, are described in Subsection B. The simulation results are augmented with an assessment of each filter's performance using LRO telemetry data, as described in Subsection C. ST quaternion data from two non-coaligned STs are available in LRO telemetry on a continuous basis. Processing both STs in a standard 6-state EKF provides a convenient means of obtaining a reasonable set of truth data for performance comparison of the proposed filtering techniques. Subsection A provides a description of the tuning parameters used in the performance evaluation.

\section{A. Filter Parameters}

Filter parameters were selected for each filter method to facilitate the performance assessment. The rationale for the values used is provided in this subsection. The Markov time constant was varied in Method \#1 to find the value that produced the minimum error variance. The value obtained was $70 \mathrm{sec}$. This seems counter-intuitive since the dominant boresight errors are centered around a $5 \mathrm{~min}$ period in the data. However, this result does make sense when considering the observability of the 6-state filter augmented with a Markov state for the measurement error. As the Markov time constant is raised the system becomes less observable; in fact it becomes entirely unobservable in the limit as the Markov state model approaches the response of a bias state. In this situation the filter cannot distinguish the gyro bias error from the ST systematic errors. Alternatively, as the time constant is lowered the Markov model response approaches the solution from a band-limited white noise model. In other words the solution from Method \#1 will approach the 6-state EKF solution in the limit.

In Method \#2 the ST systematic errors are modeled with a set of periodic basis functions with zero mean. This avoids the observability issues associated with the Markov augmentation. Periodic basis function frequencies centered around a $5 \mathrm{~min}$ period were considered a good choice for the ST periodic systematic error model for LRO, as described in Section III. Since each frequency term in the Fourier series expansion adds two states to the model, minimizing the number of terms is necessary to avoid information dilution associated with a large state vector. The filter was implemented with 3,10 , and 20 terms centered around the 5 min characteristic period. The results demonstrated that 3 terms were sufficient to estimate the short term systematic errors; additional terms did not substantially improve the solution. Thus, three fixed frequency terms with $2.5,5$, and 10 min periods were used in the-UKF implementation, resulting in a' !2-state filter. The spectral'density of the white noise sources used in the bias states associated with the basis function amplitudes in the periodic error model were tuned to provide best filter performance.

\section{B. Simulation Study}

A telemetry simulation study was conducted to assess the performance improvement of the proposed methods over the 6-state EKF. A simplified model of true rates and attitudes was generated to represent nadir attitude kinematics for approximately 5 orbits of a typical low-altitude lunar orbit with pitch rate of $0.05 \mathrm{deg} / \mathrm{sec}$. The IRU rate model included angle random walk $\left(0.3 \mathrm{arc}-\mathrm{sec} / \mathrm{sec}^{1 / 2}\right)$ and bias drift $\left(7 \mathrm{e}-5 \mathrm{arc}-\mathrm{sec} / \mathrm{sec}^{3 / 2}\right)$ noise terms. The ST error model included transverse angle ( $6.0 \mathrm{arc}-\mathrm{sec}, 3-\sigma)$ and boresight angle $(65 \mathrm{arc}-\mathrm{sec}, 3-\sigma)$ noise terms. Although two STs were simulated the filter results shown in this paper used only a single ST (STI). The two models of the ST systematic errors that were used in this simulation study are described below.

The first model implemented the ST systematic errors as a colored noise process using a $3^{\text {rd }}$ order band-pass filter. The passband was set to generate signals between $10 \mathrm{~min}$ and $2.5 \mathrm{~min}$ periods centered at a frequency associated with a 5 min period. The ST boresight and transverse errors were set to amplitudes of 28 and 4 arc-sec, 
respectively. Figure 2 shows a comparison the estimation residuals from each filter using the bandpass colored noise process to model the systematic error. The simulation duration was $35000 \mathrm{sec}$, which is equivalent to approximately five LRO orbits. These residuals were computed as the difference of each filter's attitude estimate with the simulated true attitude. Uncompensated ST systematic errors are clearly evident in the 6-state EKF results, shown in blue. Both the Markov state augmented filter (Method \#1) and the UKF with periodic measurement model (Method \#2) show significant improvement over the baseline EKF, particularly after $550 \mathrm{~min}$ when the systematic errors are largest. The UKF shows slightly better performance over that same period which demonstrates the ability of the periodic measurement model to compensate for systematic errors modeled as a random process. There are several noted exceptions to the UKF's superior performance, such as the transient at approximately $513 \mathrm{~min}$. These transients were infrequent and limited to less than $\sim 15$ arc-sec during steady-state operation.

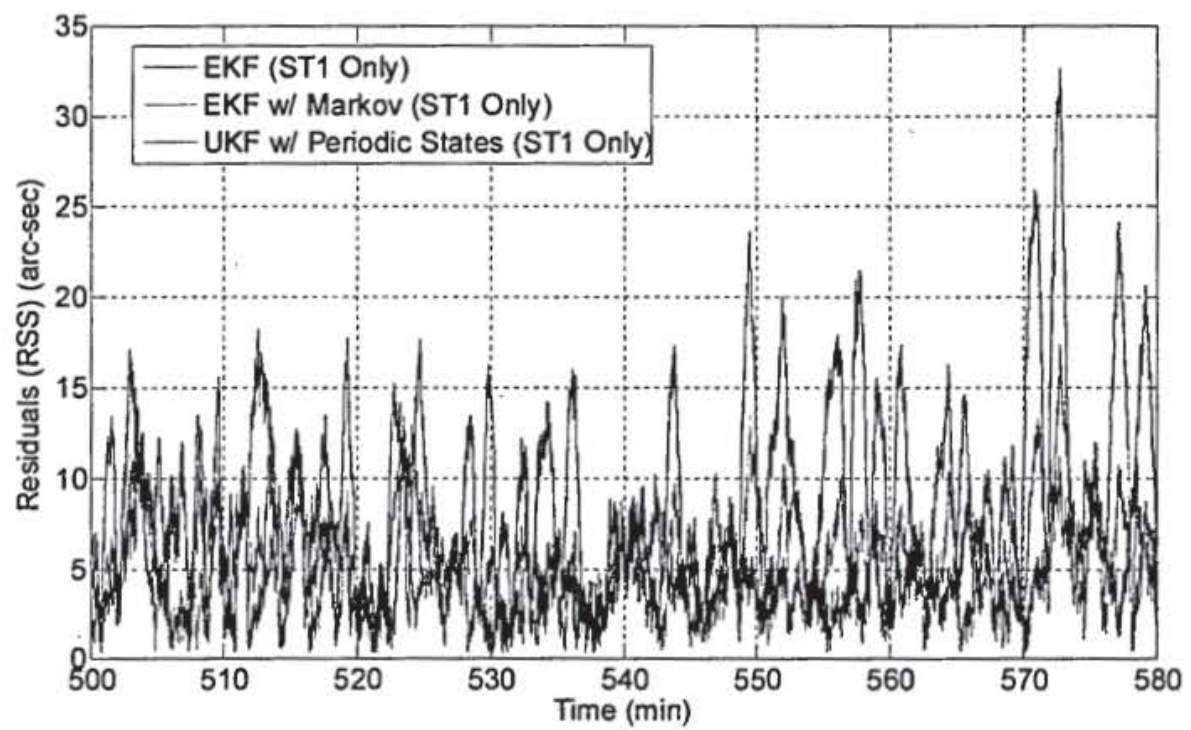

Figure 2. Filter Residuals (RSS), for EKF, Markov, and UKF, relative to True Attitude (band-pass model).

The second method modeled the ST errors in a deterministic fashion. Since the star patterns within the ST FOV repeat every orbit in a Nadir pointing situation, a periodic model provided a reasonable alternative to a random process model for evaluating realistic performance in the presence of periodic errors. In this case the ST systematic errors were comprised of sine and cosine waveforms at three fixed frequencies associated with $2.5,5$, and $10 \mathrm{~min}$ periods. These frequencies where selected for two reasons, 1) to provide an approximate model of the time-varying - nature of the systematic error associated with the star transits through ST FOV, as described in Section III, 2) to match the frequencies used in the UKF periodic measurement model to quantify the potential improvement in filter performance using this method, as well as the robustness of the Markov state augmentation to non-random errors in

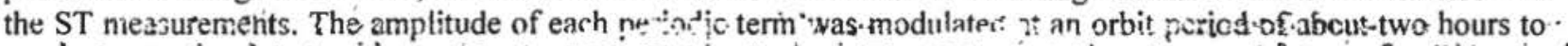
approximate the vàriations noticed in the LRO flight telemetry. The ST boresight and transverse errors were set to amplitudes of 28 and 4 arc-sec, respectively, similar to the approach used to model the ST errors in the band-pass error model. Figure 3 shows a comparison the estimation residuals from each filter using the periodic model of the ST systematic error. The simulation duration was $35000 \mathrm{sec}$, which is equivalent to approximately five LRO orbits. Uncompensated ST systematic errors are clearly evident in the 6-state EKF results, shown in blue. Both the Markov state augmented filter (Method \#1) and the UKF with periodic measurement model (Method \#2) show significant improvement over the baseline EKF, for the selected period representing steady-state filter operation. The UKF results, shown in red, demonstrate a factor of approximately two or better performance than the EKF with Markov state over this period, illustrating the potential performance gain associated with the periodic measurement model. There is one noted exception to the UKF's superior performance at approximately $568 \mathrm{~min}$. Transients of this type were infrequent and limited to less than $\sim 15$ arc-sec in the steady-state, similar to the results obtained when using the band-pass model. Comparing the results of the EKF with Markov state using the periodic ST error model, shown in Figure 2, to the results using the band-pass colored noise model, shown in Figure 3, illustrate the robustness of the Markov state implementation.' In - both cases the EKF with Markov state provides approximately a factor of two improvement over the baseline EKF. 


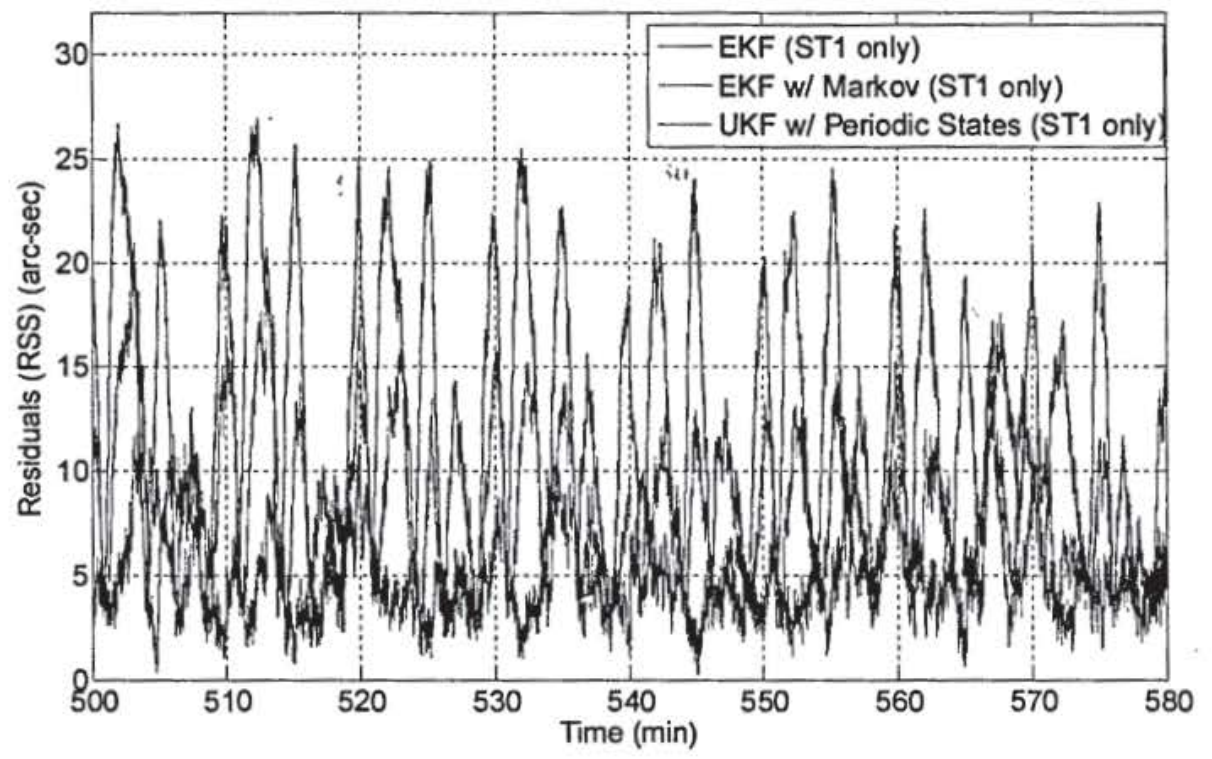

Figure 3. Filter Residuals (RSS), for EKF, Markov, and UKF, relative to True Attitude (periodic model).

\section{Filter Processing of LRO Flight Telemetry}

Filter processing of LRO flight telemetry data was used to assess the performance of the proposed attitude estimation methods, supplementing the simulation results presented in the previous section. This telemetry assessment provides a cross-validation of the simulated data estimates that utilize hueristic models in place of more precise high fidelity models of the ST error contributors.

The relative performance of the two proposed filters are assessed by processing LRO flight telemetry from a single ST and comparing against an estimated attitude obtained by processing both STs with the standard 6-state EKF. Since the FOV of each ST does not overlap the other the systematic errors associated with each ST should not be correlated with each other. Thus, the resulting attitude solutions from a 6-state filter that processes measurements from both STs simultaneously should provide a reasonable baseline for determination of the relative performance improvements from the proposed filtering methods. An analysis of single ST attitude solutions as compared against two ST filter solutions provides a validation of this approach. Figure 4 shows a comparison of attitude estimation residuals from a typical two orbit period of LRO flight data. These residuals are computed as the difference of a standard 6-state Kalman filter solution using STI measurements only to the baseline obtained from a 6-state filter processing data from both STs. Note that the estimation error has been expressed in the local ST1 reference frame (Z axis is ST boresight). As expected the error is dominant about the STI boresight. Figure 5 shows a similar EKF

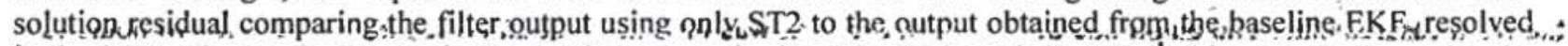
in the ST2 reference frame. Again the dominant error is about the ST boresight, ST2 in this case. Furthermore, the error in each case appears to be uncorrelated with the other. This seems to indicate that I) the ST systematic error is dominated by error about each respective ST boresight, and 2) the solution for the 6-state EKF using both STs is accurate enough to provide a good baseline for performance assessment of alternative approaches. 


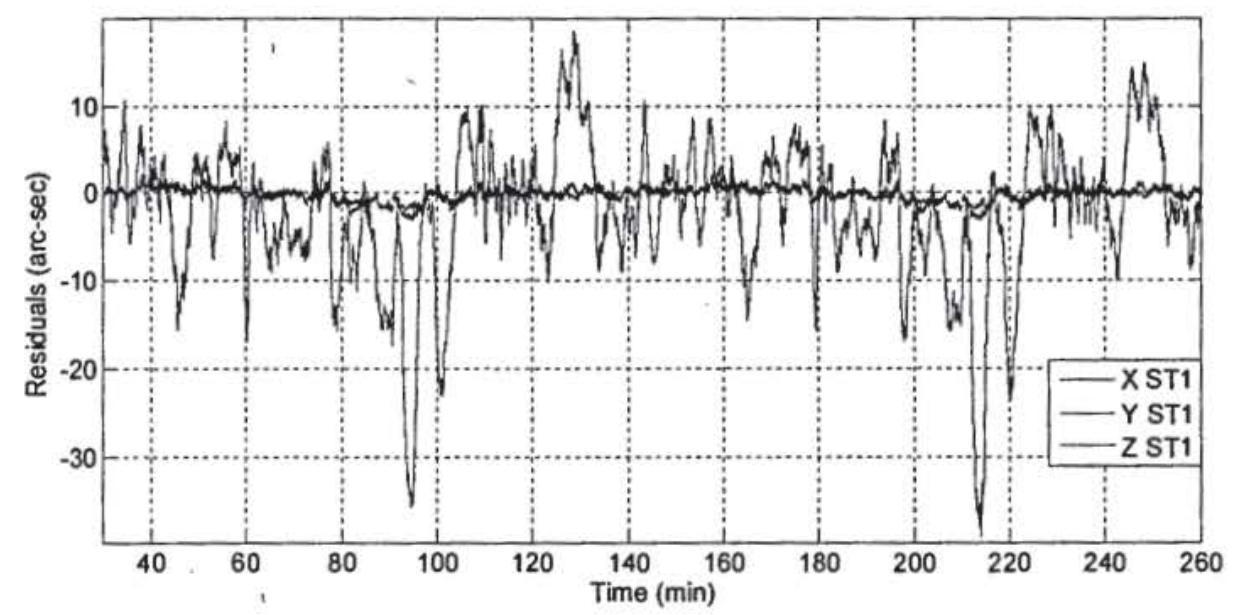

Figure 4. Filter Residuals, EKF (ST1 only) relative to EKF (Both STs), Expressed in [ST1] Frame

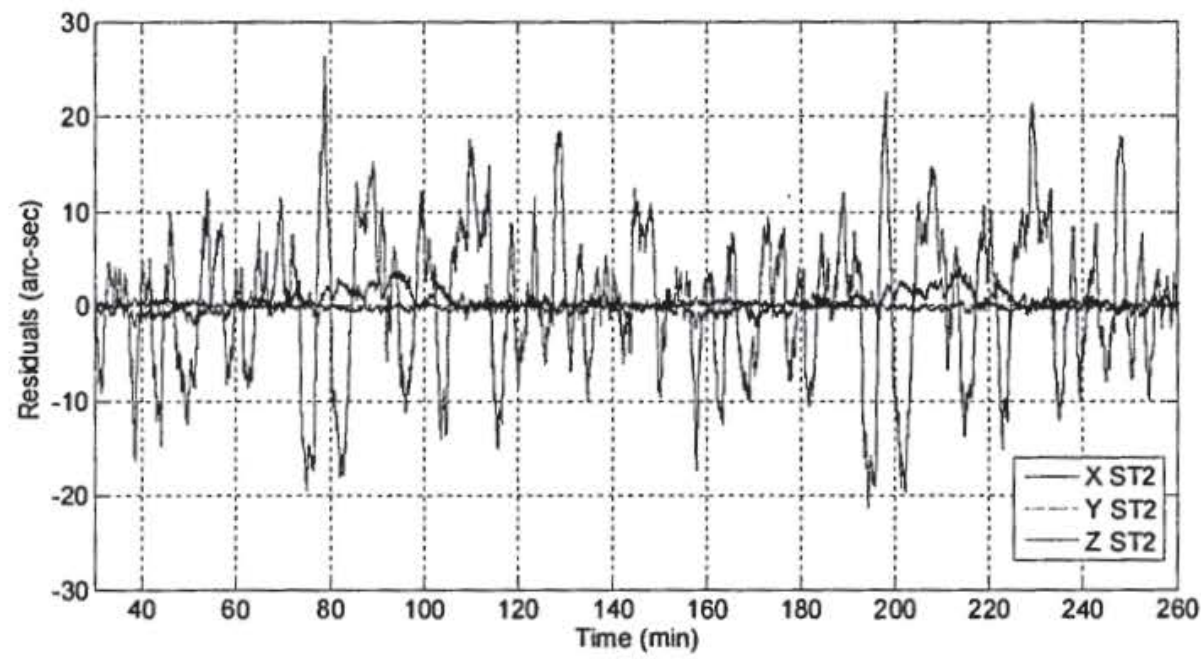

Figure 5. Filter Residuals, EKF (ST2 only) relative to EKF (Both STs), Expressed in [ST2] Frame

Each proposed filter method was evaluated by comparison against the baseline 6-state EKF solution using a representative set of LRO date. Figure 6 sho $\because 6$ the roct sure square (RSS) attitude residcals computed by differencing each proposed filter solution using only ST1 with the baseline filter using both.STs. Figure 7 shows a similar set of comparisons using ST2 data. Results of both the EKF with Markov state (Method \#1), and the UKF with periodic measurement model (Method \#2) show improvement in reducing the'error associated with the 2-10 min period variations evident in the data. The UKF outperforms the standard 6-state EKF, as well as the EKF augmented with the Markov, state. This performance improvement is more evident when considering the comparative filter results shown in Figures 8 and 9 . These plots were produced by forward-backward filtering the residuals shown in Figs. 6 and 7 using a first-order high-pass filter with a 15 min time constant to remove low frequency errors primarily attributed to thermal alignment stability within an orbit period, such as those evident in ST1 telemetry between 1130 and $1150 \mathrm{~min}$. Knowledge error stability within a 15 min time period would be of primary importance in removing any image distortion since the LRO Camera (LROC) typically processes single images during this period. Performance improvements for the UKF (Method \#2) over the baseline 6-state EKF are evident in these figures. 


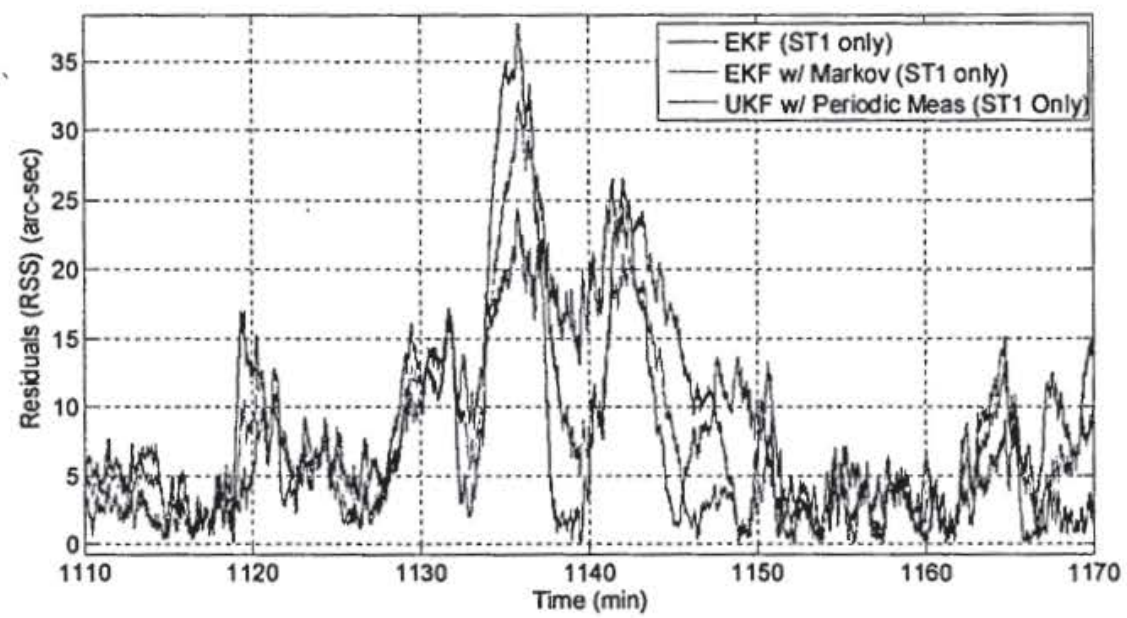

Figure 6. Filter Residuals (RSS), for EKF, Markov, and UKF models, (ST1 only) relative to EKF (both STs).

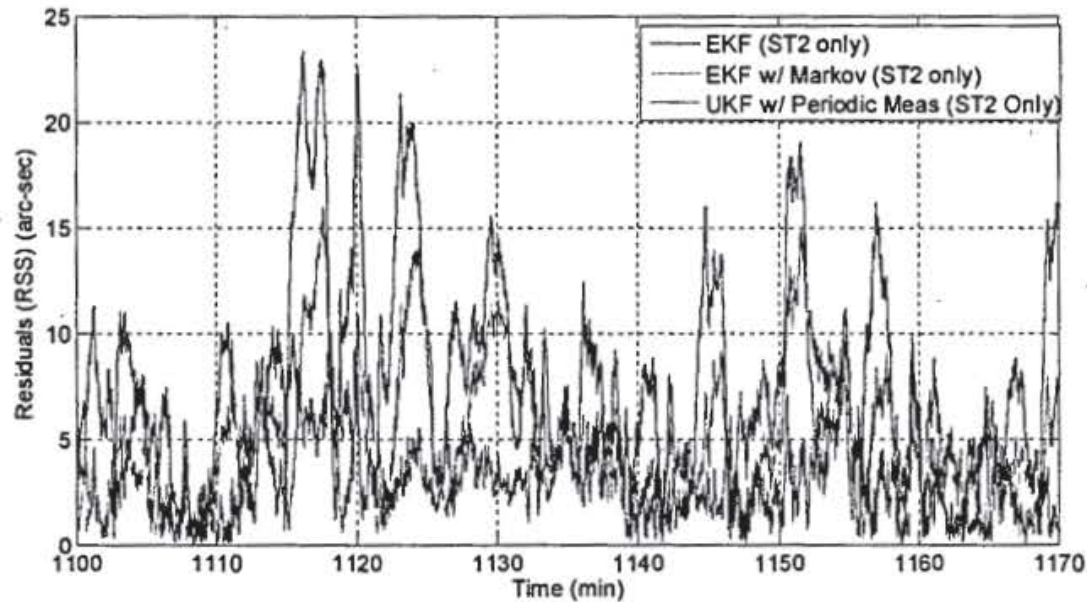

Figure 7. Filter Residuals (RSS), for EKF, Markov, and UKF models, (ST2 only) relative to EKF (both STs).

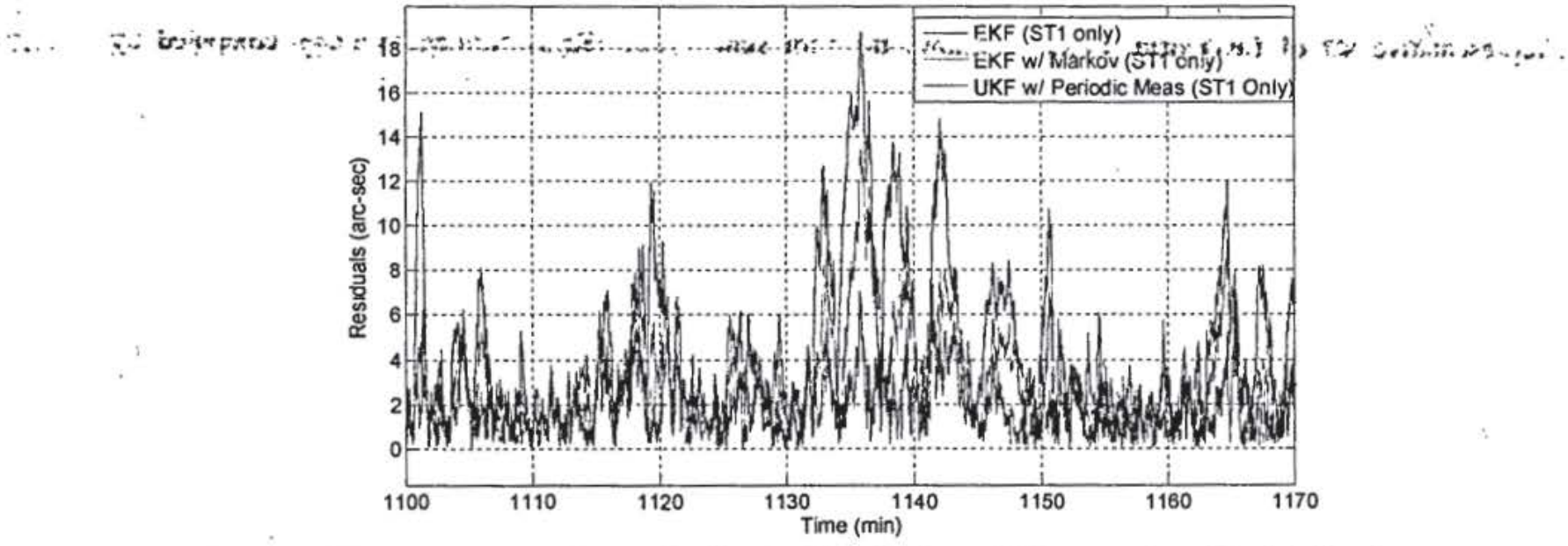

Figure 8. Filter Residuals (RSS), as in Fig. 6, with additional high-pass $\left(T_{h p}=15 \mathrm{~min}\right.$ ) filtering. 


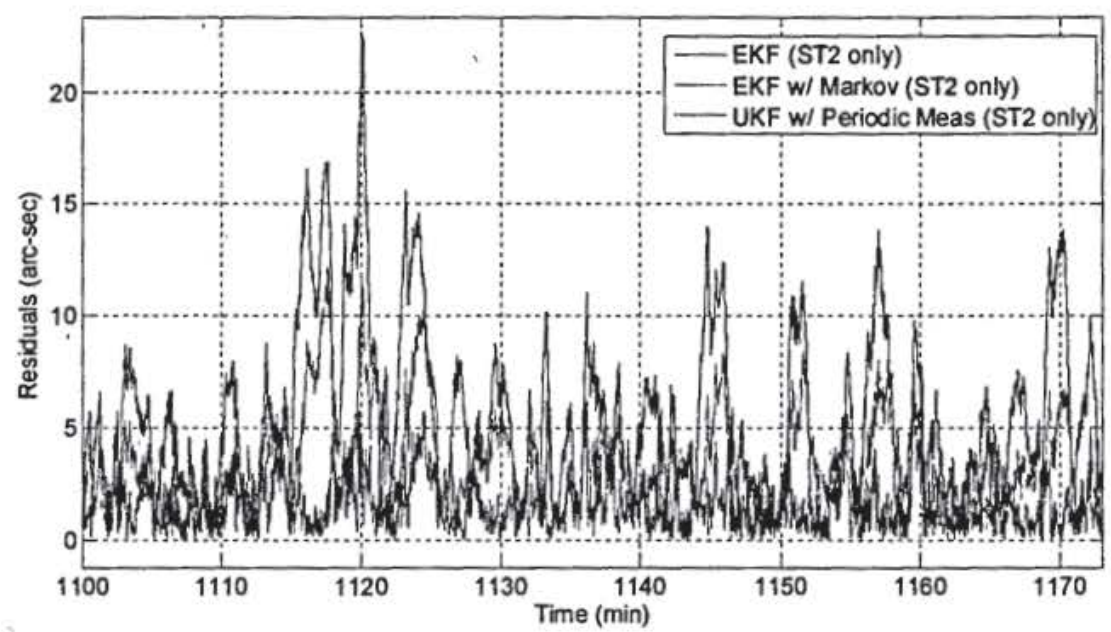

Figure 9. Filter Residuals (RSS), as in Fig. 7, with additional high-pass $\left(\mathrm{T}_{\mathrm{hp}}=15 \mathrm{~min}\right)$ filtering.

\section{Conclusion}

Systematic error in quaternion star trackers has been identified as a primary error source in spacecraft attitude estimation using a typical GNC hardware complement. Six-state attitude filtering approaches do not actively attempt to mitigate these non-white, non-Gaussian errors sources. Two attitude filter methods, one using an EKF with Markov state augmentation, and the other using a UKF with periodic measurement model, have been shown to improve the attitude estimation using both simulated data and LRO flight telemetry. The UKF with periodic basis function measurement model shows the greater improvement over the traditional 6-state EKF in reducing the effects of temporally correlated systematic errors in the ST measurements for a typical non-inertial pointing application. Further work to reduce the infrequent error transients noticed in the UKF, and to exploit the capability of the UKF to handle nonlinear state and measurement processes - perhaps with on-line adaptive estimation of the basis function parameters - is an ongoing effort.

\section{References}

1. E. J. Lefferts, F. L. Markley, and M. D. Shuster, "Kalman Filtering for Spacecraft Attitude Estimation," Journal of Guidance, Control, and Dynamics, Vol. 5, No. 5, Sept.-Oct. 1982, pp. 417-429.

2. D.R. Haley, T.E. Strikwerda, H.L. Fisher, and G.A. Heyler, "Attainable Pointing Accuracy with Star Trackers," $21^{\text {st }}$ Annual AAS Guidance and Control Conference, Breckenridge, Co., February 4-8, 1998.

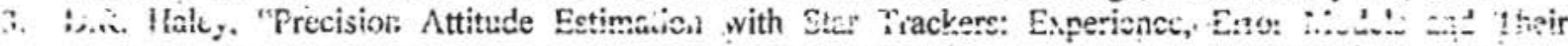
Interpretation," AAS/AIAA Astrodynamics Specialist Conference, Girdwood, Al., August 16-19, 1999.

4. Calhoun, P.C., Garrick, J.C., "Observing Mode Attitude Controller for the Lunar Reconnaissance Orbiter," 20th International Symposium on Space Flight Dynamics, Annapolis, Md., September 24-28, 2007.

5. J. L. Crassidis, F. L. Markley, Y. Cheng, "Survey of Nonlinear Attitude Estimation Methods," Journal of Guidance, Control, and Dynamics, 2007.

6. J. Sedlak, J. Hashmall, "Accurate Magnetometer/Gyroscope Attitudes Using a Filter With Correlated Sensor Noise," NASA / GSFC Flight Mechanics Symposium, 1997.

7. M. Wang, P. Y. Li, "Duty Ratio Control of a Rotary PWM Valve with Periodic Measurement Error," American Control Conference, 2009.

8. R. Van Der Merwe, "Sigma-Point Kalman Filters for Probabilistic Inference in Dynamic State-Space Models," PhD Dissertation, 2004.

9. S. J. Julier, J. K. Uhlmann, "A New Extension of the Kalman Filter to Nonlinear Systems," SPIE 1997.

10. Gelb, A. (ed.), Applied Optimal Estimation, The MIT Press, Cambridge, MA, 1974. 